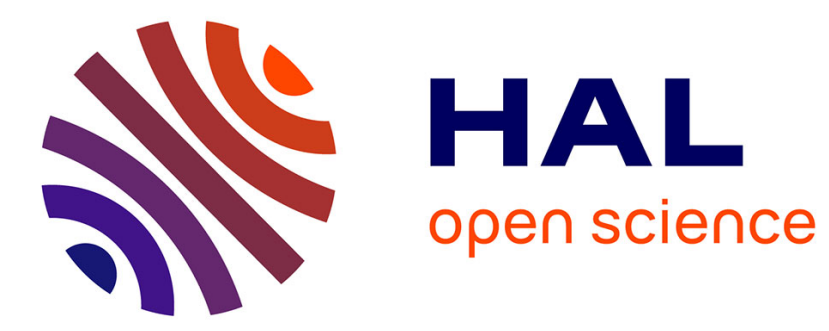

\title{
Minimal simple pairs in the 3-D cubic grid
}

Nicolas Passat, Michel Couprie, Gilles Bertrand

\section{To cite this version:}

Nicolas Passat, Michel Couprie, Gilles Bertrand. Minimal simple pairs in the 3-D cubic grid. Journal of Mathematical Imaging and Vision, 2008, 32 (3), pp.239-249. 10.1007/s10851-008-0099-9 . hal00622368

\section{HAL Id: hal-00622368 https://hal.science/hal-00622368}

Submitted on 1 Mar 2018

HAL is a multi-disciplinary open access archive for the deposit and dissemination of scientific research documents, whether they are published or not. The documents may come from teaching and research institutions in France or abroad, or from public or private research centers.
L'archive ouverte pluridisciplinaire HAL, est destinée au dépôt et à la diffusion de documents scientifiques de niveau recherche, publiés ou non, émanant des établissements d'enseignement et de recherche français ou étrangers, des laboratoires publics ou privés. 


\title{
Minimal simple pairs in the 3-D cubic grid
}

\author{
N. Passat ${ }^{(a)}$, M. Couprie ${ }^{(b)}$ and G. Bertrand ${ }^{(b)}$ \\ (a) LSIIT, UMR 7005 CNRS/ULP, Strasbourg 1 University, France \\ (b) Université Paris-Est, LABINFO-IGM, UMR CNRS 8049, A2SI-ESIEE, France \\ e-mail: passat@dpt-info.u-strasbg.fr, (m.couprie,g.bertrand)@esiee.fr
}

\begin{abstract}
Preserving topological properties of objects during thinning procedures is an important issue in the field of image analysis. This paper constitutes an introduction to the study of non-trivial simple sets in the framework of cubical 3-D complexes. A simple set has the property that the homotopy type of the object in which it lies is not changed when the set is removed. The main contribution of this paper is a characterisation of the non-trivial simple sets composed of exactly two voxels, such sets being called minimal simple pairs.
\end{abstract}

Key words: Cubical complexes, topology preservation, collapse, thinning, 3-D space.

\section{Introduction}

Topological properties are fundamental in many applications of image analysis. Topology-preserving operations, like homotopic skeletonisation, are used to transform an object while leaving unchanged its topological characteristics. In discrete grids $\left(\mathbb{Z}^{2}\right.$ or $\left.\mathbb{Z}^{3}\right)$, such a transformation can be defined and efficiently implemented thanks to the notion of simple point [18]: intuitively, a point of an object is called simple if it can be deleted from this object without altering its local topology in the vicinity of that point.

A typical topology-preserving transformation based on simple point deletion, which we call guided homotopic thinning $[11,10]$, may be described as follows. The input data consists of a set $X$ of points in the grid (called the object), and a subset $K$ of $X$ (called the constraint set). Let $X_{0}=X$. At each iteration $i$, choose a simple point $x_{i}$ in $X_{i}$ but not in $K$ according to some criterion (e.g., a priority function) and set $X_{i+1}=X_{i} \backslash\left\{x_{i}\right\}$. Continue until reaching a step $n$ such that no simple point for $X_{n}$ remains in $X_{n} \backslash K$. We call the result of this process a homotopic skeleton of $X$ constrained by $K$. Notice that, since several points may have the same priority, there may exist several homotopic skeletons for a given pair $X, K$.

The most common example of a priority function for the choice of $x_{i}$ is a distance map which associates, to each point of $X$, its distance from the boundary of $X$. In this case, the points which are closest to the boundary are chosen first, resulting in a skeleton which is "centered" in the original object. In some particular applications, the priority function may be obtained through a grey-scale image, for example when the goal is to segment objects in this image while respecting topological constraints (see e.g. $[12,25])$. In the latter case, the order in which points are considered does not rely on geometrical properties, and may be affected by noise.

One drawback of thinning algorithms that work in 
the manner we have described is that the final set $X_{n}$ is not always minimal (see discussion in Appendix D) - as one of the authors found when processing MRI images of the brain [22]. The problem here is that even though $X_{n}$ contains no simple point outside the constraint set $K$, it is still possible for $X_{n} \backslash K$ to include non-empty subsets $D$ which have the property that $X_{n}$ can be "deformed" over itself, in a sense that will be made precise in Def. 2, onto the smaller set $X_{n} \backslash D$ (so that $X_{n}$ is "homotopy equivalent" in a discrete sense to $\left.X_{n} \backslash D\right)$. A subset $D$ that has this property will be called a simple set (for $X_{n}$ ). An example of such a set is shown in the last figure of Section 4 ; if $X_{n}$ is the 14point set shown in that figure, then the set $D=\{x, y\}$ is simple for $X_{n}$. One way to address this problem would be to try to further reduce the set $X_{n}$ by finding and deleting some subset $D$ of $X_{n} \backslash K$ that is simple for $X_{n}$. (If we are able to do that, and the resulting set $X_{n} \backslash D$ contains one or more simple points that are not in $K$, then the original thinning algorithm can be used to thin the set even more.) To put this idea into practice, we need good ways of finding sets in $X_{n} \backslash K$ that are simple for $X_{n}$.

Certain classes of simple sets have been studied in the literature dedicated to parallel homotopic thinning algorithms $[23,1,14]$. In these studies, the considered simple sets are composed exclusively of simple points. In our case, the situation is radically different since our set $X_{n}$ does not contain any simple point outside of $K$. Our problem may be formulated as follows: does there exist a characterisation of certain simple sets composed of non-simple points?

We are indeed interested essentially by simple sets which are minimal, in the sense that they do not strictly include any other simple set, since it is sufficient to detect such sets in order to carry on thinning. Also, we hope that minimal simple sets have a specific structure which could make them easier to analyse.

This paper is dedicated to the study of the simplest ones among such simple sets, called simple pairs, which are those composed of two non-simple points. These minimal simple sets are the ones which are most likely to appear in practical applications (an experimental study supporting this assertion is reported in appendix A), hence the interest in understanding their structure, and proposing topological reduction algorithms based on their characterisation. After proving some properties of simple pairs, we give a character- isation of these sets which allows us to detect and remove them when performing homotopic thinning.

We shall develop this work in the framework of abstract complexes. Abstract complexes have been promoted in particular by V. Kovalevsky [19] in order to provide a sound topological basis for image analysis. In particular, in this framework we retrieve the main notions and results of digital topology, such as the notion of simple point. In order to make the paper self-contained, we recall in the next two sections some basic definitions and properties related to complexes (see also $[5,3,4]$ for more details).

\section{Cubical complexes}

Intuitively, a cubical complex may be thought of as a set of elements having various dimensions (e.g. cubes, squares, edges, vertices) glued together according to certain rules. For some illustrations of the notions defined hereafter, the reader may refer to Figure 1.

Let $\mathbb{Z}$ be the set of integers. We consider the families of sets $\mathbb{F}_{0}^{1}, \mathbb{F}_{1}^{1}$, such that $\mathbb{F}_{0}^{1}=\{\{a\} \mid a \in \mathbb{Z}\}, \mathbb{F}_{1}^{1}=$ $\{\{a, a+1\} \mid a \in \mathbb{Z}\}$. A subset $f$ of $\mathbb{Z}^{n}(n \geq 1)$ which is the Cartesian product of exactly $m$ elements of $\mathbb{F}_{1}^{1}$ and $(n-m)$ elements of $\mathbb{F}_{0}^{1}$ is called a face or an $m$-face of $\mathbb{Z}^{n}, m$ is the dimension of $f$, and we write $\operatorname{dim}(f)=m$.

We denote by $\mathbb{F}^{n}$ the set composed of all $m$-faces of $\mathbb{Z}^{n}$ ( $m=0$ to $n$ ). An $m$-face of $\mathbb{Z}^{n}$ is called a point if $m=0$, a (unit) interval if $m=1$, a (unit) square if $m=2$, a (unit) cube if $m=3$. In the sequel, we will focus on $\mathbb{F}^{3}$.

Let $f$ be a face in $\mathbb{F}^{3}$. We set $\hat{f}=\left\{g \in \mathbb{F}^{3} \mid g \subseteq f\right\}$, and $\hat{f}^{*}=\hat{f} \backslash\{f\}$. Any $g \in \hat{f}$ is a face of $f$, and any $g \in \hat{f}^{*}$ is a proper face of $f$. If $F$ is a finite set of faces of $\mathbb{F}^{3}$, we write $F^{-}=\bigcup\{\hat{f} \mid f \in F\}, F^{-}$is the closure of $F$.

A set $F$ of faces of $\mathbb{F}^{3}$ is a cell or an $m$-cell if there exists an $m$-face $f \in F$, such that $F=\hat{f}$. The boundary of a cell $\hat{f}$ is the set $\hat{f}^{*}$.

A finite set $F$ of faces of $\mathbb{F}^{3}$ is a complex (in $\mathbb{F}^{3}$ ) if for any $f \in F$, we have $\hat{f} \subseteq F$, i.e., if $F=F^{-}$. Any subset $G$ of a complex $F$ which is also a complex is a subcomplex of $F$. If $G$ is a subcomplex of $F$, we write $G \preceq F$. If $F$ is a complex in $\mathbb{F}^{3}$, we also write $F \preceq \mathbb{F}^{3}$.

A face $f \in F$ is a facet of $F$ if there is no $g \in F$ such that $f \in \hat{g}^{*}$. We denote by $F^{+}$the set composed 


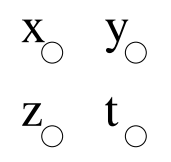

(a)

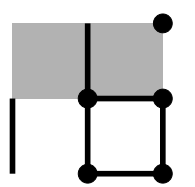

(c)

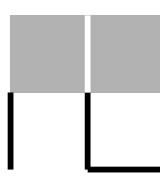

(d) (b)

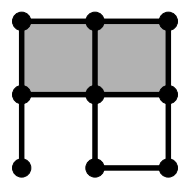

(e)
Figure 1. (a) Four points $x, y, z, t$ of $\mathbb{F}^{2}$ such that $\{x, y, z, t\}$ is a 2-face. (b) A representation of the set of faces $\{\{x, y, z, t\},\{x, y\},\{z\}\}$. (c) A set $F$ of faces in $\mathbb{F}^{2}$ : we see that $F$ is not a complex. (d) The set $F^{+}$, composed by the facets of $F$. (e) The set $F^{-}$, i.e. the closure of $F$, which is a complex.

of all facets of $F$.

Observe that $\left(F^{+}\right)^{-}=F^{-}$and thus, that $\left(F^{+}\right)^{-}=$ $F$ whenever $F$ is a complex.

The dimension of a non-empty complex $F$ in $\mathbb{F}^{3}$ is defined by $\operatorname{dim}(F)=\max \left\{\operatorname{dim}(f) \mid f \in F^{+}\right\}$. We say that $F$ is an m-complex if $\operatorname{dim}(F)=m$.

Two distinct faces $f$ and $g$ of $\mathbb{F}^{3}$ are adjacent if $f \cap g \neq \emptyset$. Let $F \preceq \mathbb{F}^{3}$ be a non-empty complex. A sequence $\left(f_{i}\right)_{i=0}^{\ell}$ of faces of $F$ is a path in $F$ (from $f_{0}$ to $\left.f_{\ell}\right)$ if $f_{i}$ and $f_{i+1}$ are adjacent, for all $i \in[0, \ell-$ $1]$. We say that $F$ is connected if, for any two faces $f, g$ in $F$, there is a path from $f$ to $g$ in $F$. We say that $G$ is a connected component of $F$ if $G \preceq F, G$ is connected and if $G$ is maximal for these two properties (i.e., we have $H=G$ whenever $G \preceq H \preceq F$ and $H$ is connected). We denote by $C[F]$ the set of all the connected components of $F$. We set $C[\emptyset]=\emptyset$.

\section{Topology-preserving operations}

\section{Collapsing}

Collapsing is a well-known operation of topology that preserves homotopy type. Let $F$ be a complex in $\mathbb{F}^{3}$ and let $f \in F$. If $g \in \hat{f}^{*}$ is such that $f$ is the only face of $F$ which strictly includes $g$, then we say that the pair $(f, g)$ is a free pair for $F$. Note that this implies $f \in F^{+}$and $\operatorname{dim}(g)=\operatorname{dim}(f)-1$. If $(f, g)$ is a free pair for $F$, the complex $F \backslash\{f, g\}$ is an elementary collapse of $F$.
Let $F, G$ be two complexes. We say that $F$ collapses onto $G$ if there exists a collapse sequence from $F$ to $G$, i.e., a sequence of complexes $\left\langle F_{0}, \ldots, F_{\ell}\right\rangle$ such that $F_{0}=F, F_{\ell}=G$, and $F_{i}$ is an elementary collapse of $F_{i-1}, i=1, \ldots, \ell$. An example of a collapse sequence is shown in Figure 2.

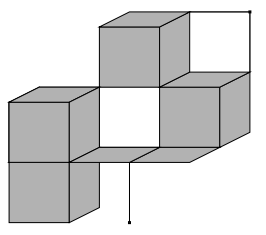

(a)

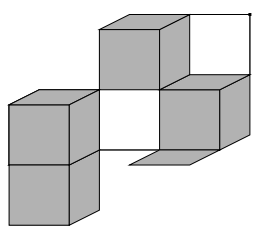

(c)

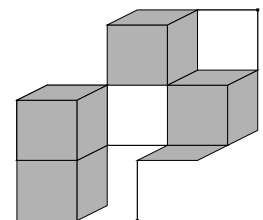

(b)

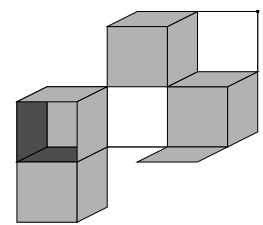

(d)
Figure 2. (a) A complex $F \preceq \mathbb{F}^{3}$. (b,c,d) Three subcomplexes of $F$ for which $\langle a, b, c, d\rangle$ is a collapse sequence.

Let $F, G$ be two complexes. Let $H$ be such that $F \cap$ $G \preceq H \preceq G$, and let $f, g \in H \backslash F$. The pair $(f, g)$ is a free pair for $F \cup H$ if and only if $(f, g)$ is a free pair for $H$. Thus, by induction, we have the following proposition.

Proposition 1 ([2]) Let $F, G \preceq \mathbb{F}^{3}$. The complex $F \cup G$ collapses onto $F$ if and only if $G$ collapses onto $F \cap G$.

\section{Topological invariants}

Let $F$ be a complex in $\mathbb{F}^{3}$, and let us denote by $n_{i}$ the number of $i$-faces of $F, i=0, \ldots, 3$. The Euler characteristic of $F$, written $\chi(F)$, is defined by $\chi(F)=$ $n_{0}-n_{1}+n_{2}-n_{3}$. The Euler characteristic is a wellknown topological invariant, and it is easy to see that the collapse operation preserves it. This invariant will play an essential role in the proofs of this paper.

Let $F, G \preceq \mathbb{F}^{3}$. A fundamental and well-known property of the Euler characteristic, analogous to the socalled inclusion-exclusion principle in set theory, is the following: $\chi(F \cup G)=\chi(F)+\chi(G)-\chi(F \cap G)$.

The Euler-Poincaré formula shows a deep link between the Euler characteristic and the Betti numbers, 
which are topological invariants defined from the homology groups ${ }^{1}$ of a complex. Intuitively, the Betti numbers $b_{0}, b_{1}, b_{2}$ correspond respectively to the number of connected components, tunnels and cavities of $F$. The Euler-Poincare formula, in the case of a complex $F$ in $\mathbb{F}^{3}$, states that $\chi(F)=b_{0}-b_{1}+b_{2}$. Betti numbers are also preserved by collapse.

\section{Simplicity}

Intuitively, a part $G$ of a complex $F$ is called simple if there is a topology-preserving deformation of $F$ over itself onto the relative complement of $G$ in $F$. We recall here a definition of simplicity (see [2]) based on the collapse operation, which can be seen as a discrete counterpart of the one given by T.Y. Kong [17].

Definition 2 Let $G \preceq F \preceq \mathbb{F}^{3}$. We set $F \oslash G=\left(F^{+} \backslash\right.$ $\left.G^{+}\right)^{-}$. The set $F \odot G$ is a complex which is the detachment of $G$ from $F$. We say that $G$ is simple for $F$ if $F$ collapses onto $F \otimes G$. Such a subcomplex $G$ is called $a$ simple subcomplex of $F$ or a simple set for $F$.

It has to be noticed that this definition of simple set is different (and more general) than the one proposed in articles on minimal non-simple sets such as $[20,16,14]$, where simple sets are iteratively composed of simple points. For example, if a minimal simple set in the sense of this paper (see Definition 4 below) contains more than one voxel, then its set of voxels will not be simple in the sense of $[20,16,14]$.

Let $G \preceq F \preceq \mathbb{F}^{3}$. The attachment of $G$ to $F$ is the complex defined by $\operatorname{Att}(G, F)=G \cap(F \odot G)$. This notion of attachment leads to a local characterisation of simple sets: Proposition 3 is a special case of Proposition 1 as $(F \odot G) \cup G=F$.

Proposition 3 Let $G \preceq F \preceq \mathbb{F}^{3}$. The complex $G$ is simple for $F$ if and only if $G$ collapses onto Att $(G, F)$.

\section{Minimal simple pairs in $\mathbb{F}^{3}$}

In the image processing literature, a digital image is often considered as a set of pixels in 2-D or voxels in 3-D. A voxel is an elementary cube, thus an easy correspondence can be made between this classical view and the framework of cubical complexes. In the

\footnotetext{
1 An introduction to homology theory can be found e.g. in [15].
}

sequel of the paper, we use the term voxel to mean a 3-cell. If a complex $F \preceq \mathbb{F}^{3}$ is a union of voxels, we write $F \sqsubseteq \mathbb{F}^{3}$. If $F, G \sqsubseteq \mathbb{F}^{3}$ and $G \preceq F$, then we write $G \sqsubseteq F$. From now on, we consider only complexes which are unions of voxels.

Notice that if $F \sqsubseteq \mathbb{F}^{3}$ and if $\hat{f}$ is a voxel of $F$, then $F \ominus \hat{f} \sqsubseteq \mathbb{F}^{3}$. There is indeed an equivalence between the operation on complexes that consists in removing (by detachment) a simple voxel, and the removal of a 26-simple voxel in the framework of digital topology (see $[16,4])$.

Definition 4 Let $G \sqsubseteq F, G \neq \emptyset$. The subcomplex $G$ is a minimal simple set (for $F$ ) if $G$ is a simple set for $F$ and $G$ is minimal with respect to the relation $\sqsubseteq$ (i.e. $H=G$ whenever $H \sqsubseteq G$ and $H$ is a non-empty simple set for $F$ ).

As stated in the introduction, the minimal simple sets which are most likely to appear in thinning processes are those which are composed of only two voxels. This statement is supported by an experimental study reported in Appendix A. In this paper, we will concentrate on this particular - but very frequent case, and provide a definition, some properties and a characterisation of these sets.

Definition 5 Let $P$ be a minimal simple set for $F$ which is composed of two voxels. Then we call $P$ a minimal simple pair, or MSP (for $F$ ).

Observe that, if a voxel is a simple cell for $F$, then it is also a (minimal) simple set for $F$. Thus, any minimal simple set which contains strictly more than one voxel cannot contain any simple voxel. In particular, if $P$ is a simple set which contains only two voxels, then $P$ is an MSP if and only if it does not contain any simple voxel.

Before beginning the study of MSPs (next section), let us show an example of such a configuration. Consider the complex $F$ depicted in Figure 3a. Another representation of this object is shown in Figure $3 b$, where each cube (voxel) is represented by a black dot. It can easily be seen that the complex $F$ is connected and has no cavity and no tunnel; furthermore it can be reduced to a single voxel by iterative deletion of simple voxels. Let us now concentrate on the set formed by the two voxels $x$ and $y$.

In Figure 3c, we can see that removing $x$ from $F$ creates a tunnel. Thus $x$ is not a simple voxel. The same can be said about $y$ (see Figure $3 \mathrm{~d}$ ). But if both $x$ and $y$ are removed (see Figure $3 \mathrm{e}$ ), then we see that we 


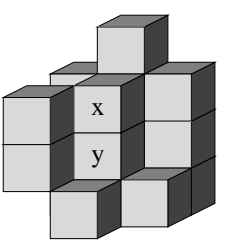

(a)

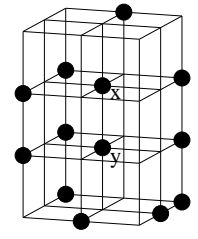

(b)

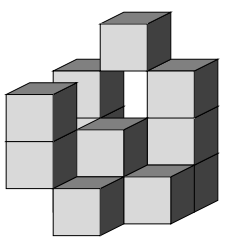

(c)

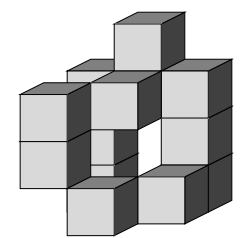

(d)

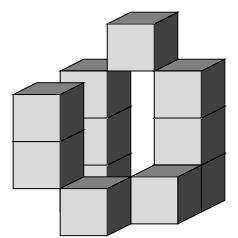

(e)
Figure 3. Example of an MSP (voxels $x$ and $y$ ). (a,b): Two representations of the same complex $F$. (c,d,e): Effect of removing either $x, y$ or both (see text).

obtain a complex $G$ which has no tunnel. It is easily verified that the union of the cells $x$ and $y$ is in fact a simple subcomplex of $F$, so that it is an MSP for $F$. This can also be deduced from Proposition 15 below.

Of course, the complex $F$ of Figure 3 a contains simple voxels (on its border). In Figure 4, we show that the same configuration can appear in a complex $H$ which has no simple voxel. Thus, $H$ can be homotopically reduced by deletion of the simple pair $\{x, y\}$. The obtained result could then be further reduced to a singleton set by iterative simple voxel removal. Notice that $H$ is made of only 32 voxels; it has been found by using a randomised homotopic thinning algorithm, starting from a 5 voxel-width cube.

There exist examples that contain fewer points; the smallest one we were able to build so far is composed of only 14 voxels: it has some tunnels (see Figure 5). We conjecture that 14 is the smallest possible size for a set that consists entirely of non-simple voxels but which contains an MSP.

\section{Some properties of minimal simple pairs}

We begin this section by quoting a characterisation of 3-D simple voxels proposed by Kong in [17] (see also [9]), which is equivalent to the following theorem for principal subcomplexes of $\mathbb{F}^{3}$; this characterisation
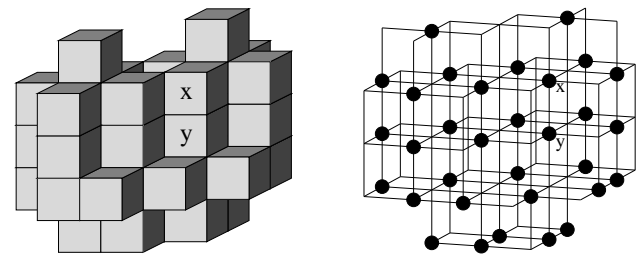

Figure 4. Left: a complex $H$ composed of non-simple voxels and which contains an MSP. Right: another representation of $H$. The subset $\{x, y\}$ is an MSP for $H$ (the removal of $\{x, y\}$ from $H$ will not alter its topology).
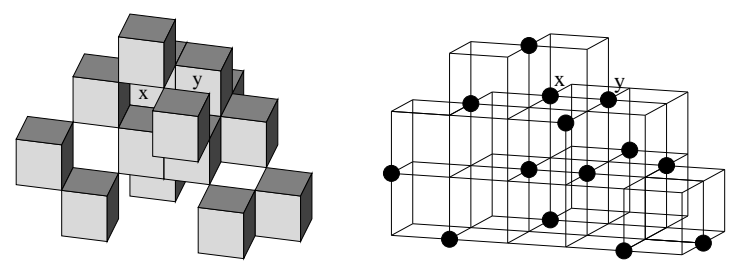

Figure 5. Left: a set of 14 non-simple voxels which contains an MSP $\{x, y\}$. It has four tunnels. Right: another representation of the same set.

will be used in the sequel. Recall that $|C[X]|$ denotes the number of connected components of $X$.

Theorem 6 (Adapted from Kong [17])

Let $F \sqsubseteq \mathbb{F}^{3}$. Let $g \in F^{+}$. Then $\hat{g}$ is a simple voxel for $F$ if and only if $|C[\operatorname{Att}(\hat{g}, F)]|=1$ and $\chi(\operatorname{Att}(\hat{g}, F))=1$.

We are now ready to state some results regarding the structure of MSPs. First of all, even though a simple set need not be connected, any MSP is connected:

Proposition 7 Let $P \sqsubseteq F$ be an MSP for $F$. Then:

$$
|C[P]|=1 .
$$

See Appendix $\mathrm{C}$ for a proof of this proposition, as well as proofs of Propositions 8 and 9, Lemma 12, and Proposition 15 below.

As discussed before, the voxels constituting an MSP cannot be simple voxels. Intuitively, the attachment of a non-simple voxel $\hat{f}$ can either:

i) be empty (isolated voxel);

ii) be equal to the boundary of $\hat{f}$ (interior voxel);

iii) be disconnected;

iv) have at least one tunnel.

Notice that iii) and iv) are not exclusive: the attachment of a non-simple voxel can both be disconnected and contain tunnels.

We will see that some of these cases cannot appear in an MSP. First, we prove that i) and iii) cannot hold for such a voxel, i.e., the attachment of a voxel in an 
MSP is non-empty and connected.

Proposition 8 Let $P \sqsubseteq F$ be an MSP for $F$. Then:

$$
\forall g \in P^{+},|C[\operatorname{Att}(\hat{g}, F)]|=1 .
$$

Then, with the next proposition, we show that ii) cannot hold, hence, the attachment to $F$ of any voxel $\hat{g}$ in an MSP has no cavity.

Proposition 9 Let $P \sqsubseteq F$ be an MSP for $F$. Then:

$$
\forall g \in P^{+}, \operatorname{Att}(\hat{g}, F) \neq \hat{g}^{*} .
$$

Recall that, according to the Euler-Poincaré formula, $\chi(\operatorname{Att}(\hat{g}, F))=b_{0}-b_{1}+b_{2}$, where $b_{0}\left(\right.$ resp. $\left.b_{2}\right)$ is the number of connected components (resp. cavities) of $\operatorname{Att}(\hat{g}, F)$. From the two previous propositions, we have $b_{0}=1$ and $b_{2}=0$. The Betti number $b_{1}$, which represents the number of tunnels, is nonnegative. Thus, we have $\chi(\operatorname{Att}(\hat{g}, F))=1-b_{1} \leq 1$. But from Theorem 6 and Proposition 8 we must have $\chi(\operatorname{Att}(\hat{g}, F)) \neq 1$, otherwise $\hat{g}$ would be a simple voxel. This proves the following proposition, which (with Proposition 8 and Proposition 9) implies that the attachment to $F$ of any voxel in an MSP has at least one tunnel.

Proposition 10 Let $P \sqsubseteq F$ be an MSP for $F$. Then:

$$
\forall g \in P^{+}, \chi(\operatorname{Att}(\hat{g}, F)) \leq 0 .
$$

From Proposition 7, we know that an MSP is necessarily connected. The following proposition tells us more about the intersection of the two voxels which compose any MSP.

Proposition 11 Let $P \sqsubseteq F$ be an MSP for $F$, and let $\hat{g}_{1}, \hat{g}_{2}$ be the two voxels of $P$. Then, $g_{1} \cap g_{2}$ is a 2-face.

This proposition is an easy consequence of the following lemma: it may be seen that Lemma 12 implies that the intersection of $\operatorname{Att}(P, F)$ with $\hat{g}_{1} \cap \hat{g}_{2}$ has at least three connected components. This is possible only when $\operatorname{dim}\left(g_{1} \cap g_{2}\right)=2$.

Lemma 12 Let $P \sqsubseteq F$ be an MSP for $F$, and let $\hat{g}_{1}, \hat{g}_{2}$ be the two voxels of $P$. Then:

$$
\chi\left(\operatorname{Att}(P, F) \cap \hat{g_{1}} \cap \hat{g_{2}}\right) \geq 3 .
$$

To illustrate the above properties, let us consider the attachment of the pair $P=\{x, y\}$ of Figure $3 \mathrm{a}$, which is displayed in Figure 6a, and the attachment of $x$ (resp. $y$ ) displayed in Figure 6b (resp. 6c). We can see in particular that the intersection of $\operatorname{Att}(P, F)$ with $x \cap y$

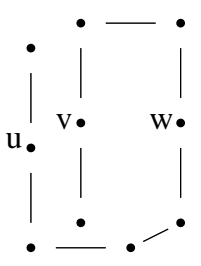

(a)

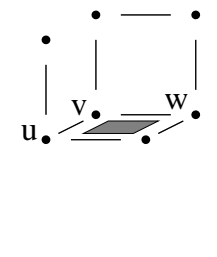

(b)

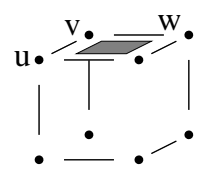

(c)
Figure 6. Attachments of configurations of Figure 3. (a): Attachment of $\{x, y\}$. (b): Attachment of $x$. (c): Attachment of $y$.

is indeed composed of three connected components (the 0 -cells $\hat{u}, \hat{v}$ and $\hat{w}$ ), as implied by Lemma 12 .

The two following propositions are necessary conditions for an MSP (similar to the conditions of Theorem 6 which characterise simple voxels).

From Proposition 3, $P$ collapses onto $\operatorname{Att}(P, F)$ whenever $P$ is an MSP. From Proposition 7, $|C[P]|=$ 1 , and since collapsing preserves the number of connected components, $|C[P]|=|C[\operatorname{Att}(P, F)]|$. Consequently we have the following.

Proposition 13 Let $P \sqsubseteq F$ be an MSP for $F$. Then:

$$
|C[\operatorname{Att}(P, F)]|=1 .
$$

Since $\chi(P)=1$, and since collapsing preserves the Euler characteristic, the following proposition is also straightforward.

Proposition 14 Let $P \sqsubseteq F$ be an MSP for $F$. Then:

$$
\chi(\operatorname{Att}(P, F))=1 \text {. }
$$

Our final proposition, and the main result of this paper, is that the properties stated in Propositions 8, $10,11,13$, and 14 above constitute a necessary and sufficient characterisation of minimal simple pairs.

Proposition 15 Let $P \sqsubseteq F$ be a pair. Then $P$ is an MSP for $F$ if and only if all the following conditions hold:

the intersection of the voxels of $P$ is a 2-cell,

$$
\begin{aligned}
\forall g \in P^{+},|C[\operatorname{Att}(\hat{g}, F)]| & =1, \\
\forall g \in P^{+}, \chi(\operatorname{Att}(\hat{g}, F)) & \leq 0, \\
|C[\operatorname{Att}(P, F)]| & =1, \\
\chi(\operatorname{Att}(P, F)) & =1 .
\end{aligned}
$$

Remark 16 Our proof of Proposition 15 (see Appendix C) will show that (1), (3), (4), and (5) are sufficient to characterise an MSP; condition (2) is a con- 
sequence of the other four conditions. It can also be shown that, if $P$ is a pair of non-simple voxels, then $P$ is an MSP for $F$ if and only if conditions (4) and (5) both hold. Indeed, if the elements of $P$ are non-simple, and (4) and (5) both hold, then arguments along the lines of our justification of Propositions 8, 10, and 11 will establish that (2), (3), and (1) also hold, so that $P$ is an MSP. This gives us a characterisation of MSPs that is similar to the characterisation of simple voxels in Theorem 6.

\section{Conclusion}

Skeletons produced by 3D thinning procedures which only delete simple voxels (or simple points) are not always minimal. The detection of MSPs, which may be done thanks to the characterisation (Proposition 15) given above, allows homotopic thinning procedures to break non-minimal objects that contain no simple voxels. Experimental results reported in Appendix A provide a quantitative evaluation of the gain in terms of reduction that can be obtained by considering MSPs in addition to simple points.

Moreover, the problems of searching for MSPs and searching for simple voxels have the same asymptotic algorithmic complexity (both being linear with respect to the number of facets of the processed complex). Consequently, it is possible to create new thinning procedures based on the detachment of both simple voxels and pairs (such as the one proposed in Appendix A) and whose runtimes have the same order of growth as the runtimes of thinning procedures that are based only on simple voxels. Such new algorithms would be able to produce skeletons that have fewer points than those produced by current algorithms.

\section{Acknowledgements}

We are grateful to the anonymous reviewers for their constructive comments, which led to significant improvement of the quality of this article.

\section{References}

[1] G. Bertrand. On P-simple points. Comptes Rendus de l'Académie des Sciences, Série Math., I(321):1077-1084, 1995.

[2] G. Bertrand. On critical kernels. Comptes Rendus de l'Académie des Sciences, Série Math., I(345):363-367, 2007.

[3] G. Bertrand and M. Couprie. New 2D parallel thinning algorithms based on critical kernels. In IWCIA, volume 4040 of $L N C S$, pages 45-59. Springer, 2006.

[4] G. Bertrand and M. Couprie. A new 3D parallel thinning scheme based on critical kernels. In $D G C I$, volume 4245 of LNCS, pages 580-591. Springer, 2006.

[5] G. Bertrand and M. Couprie. Two-dimensional thinning algorithms based on critical kernels. Journal of Mathematical Imaging and Vision, to appear, 2008.

[6] R.H. Bing. Some aspects of the topology of 3-manifolds related to the Poincaré conjecture. Lectures on Modern Mathematics II, pages 93-128, 1964.

[7] J. Chaussard. Personnal communication. 2007.

[8] D.R.J. Chillingworth. Collapsing three-dimensional convex polyhedra. Proceedings of the Cambridge Philosophical Society, 63:353-357, 1967.

[9] M. Couprie and G. Bertrand. New characterizations of simple points in 2D, 3D and 4D discrete spaces. Technical Report IGM2007-07, Université de Marne-la-Vallée, 2007. Submitted.

[10] M. Couprie, D. Coeurjolly, and R. Zrour. Discrete bisector function and Euclidean skeleton in 2D and 3D. Image and Vision Computing, 25(10):1543-1556, 2007.

[11] E.R. Davies and A.P.N. Plummer. Thinning algorithms: a critique and a new methodology. Pattern Recognition, 14(16):53-63, 1981.

[12] P. Dokládal, C. Lohou, L. Perroton, and G. Bertrand. Liver blood vessels extraction by a 3-D topological approach. In MICCAI, volume 1679 of LNCS, pages 98-105. Springer, 1999.

[13] S. Fourey and R. Malgouyres. A concise characterization of 3D simple points. Discrete Applied Mathematics, 125(1):5980, 2003.

[14] C.-J. Gau and T.Y. Kong. Minimal non-simple sets in 4D binary pictures. Graphical Models, 65(1-3):112-130, 2003.

[15] P. Giblin. Graphs, surfaces and homology. Chapman and Hall, 1981.

[16] T. Yung Kong. On topology preservation in 2-D and 3-D thinning. International Journal on Pattern Recognition and Artificial Intelligence, 9(5):813-844, 1995.

[17] T. Yung Kong. Topology-preserving deletion of 1's from 2-, 3- and 4-dimensional binary images. In $D G C I$, volume 1347 of LNCS, pages 3-18. Springer, 1997. 
[18] T. Yung Kong and A. Rosenfeld. Digital topology: introduction and survey. Computer Vision, Graphics and Image Processing, 48(3):357-393, 1989.

[19] V.A. Kovalevsky. Finite topology as applied to image analysis. Computer Vision, Graphics and Image Processing, 46(2):141161, 1989.

[20] C.M. Ma. On topology preservation in 3D thinning. Computer Vision, Graphics and Image Processing, 59(3):328-339, 1994.

[21] D.G. Morgenthaler. Three-dimensional simple points: serial erosion, parallel thinning, and skeletonization. Technical Report TR-1005, University of Maryland, 1981.

[22] N. Passat, C. Ronse, J. Baruthio, J.-P. Armspach, M. Bosc, and J. Foucher. Using multimodal MR data for segmentation and topology recovery of the cerebral superficial venous tree. In $I S V C$, volume 3804 of $L N C S$, pages 60-67. Springer, 2005.

[23] C. Ronse. Minimal test patterns for connectivity preservation in parallel thinning algorithms for binary digital images. Discrete Applied Mathematics, 21(1):67-79, 1988.

[24] A. Rosenfeld. Connectivity in digital pictures. Journal of the Association for Computer Machinery, 17(1):146-160, 1970.

[25] F. Ségonne. Segmentation of Medical Images under Topological Constraints. PhD thesis, MIT, 2005.

\section{Appendix A: Experimental study}

We applied two homotopic reduction algorithms to cubical objects of varying sizes, ranging from 1000 voxels $(10 \times 10 \times 10$ cube $)$ to about 200000 voxels $(59 \times 59 \times 59$ cube $)$. The first algorithm was based on simple point removal (see Algorithm 1 below); the second one was based on both simple point and MSP removal (see Algorithm 2). In both algorithms, the removal order depended on a random priority function $p$.

In Figure 7, we can observe and compare the effectiveness of both reduction methods, that is, the number of cases (computed from 1000 experiments for the same object size) where the thinning could be performed until only a single voxel remained. These results show that using the notion of MSP enables us to significantly improve the effectiveness of topologypreserving reduction procedures.

We also observed that, among the objects output by Algorithm 1 which consisted of more than one voxel, roughly 92 percent contained at least one MSP. This proportion did not significantly vary with the size of the original object.

\section{Appendix B: Preliminary properties}
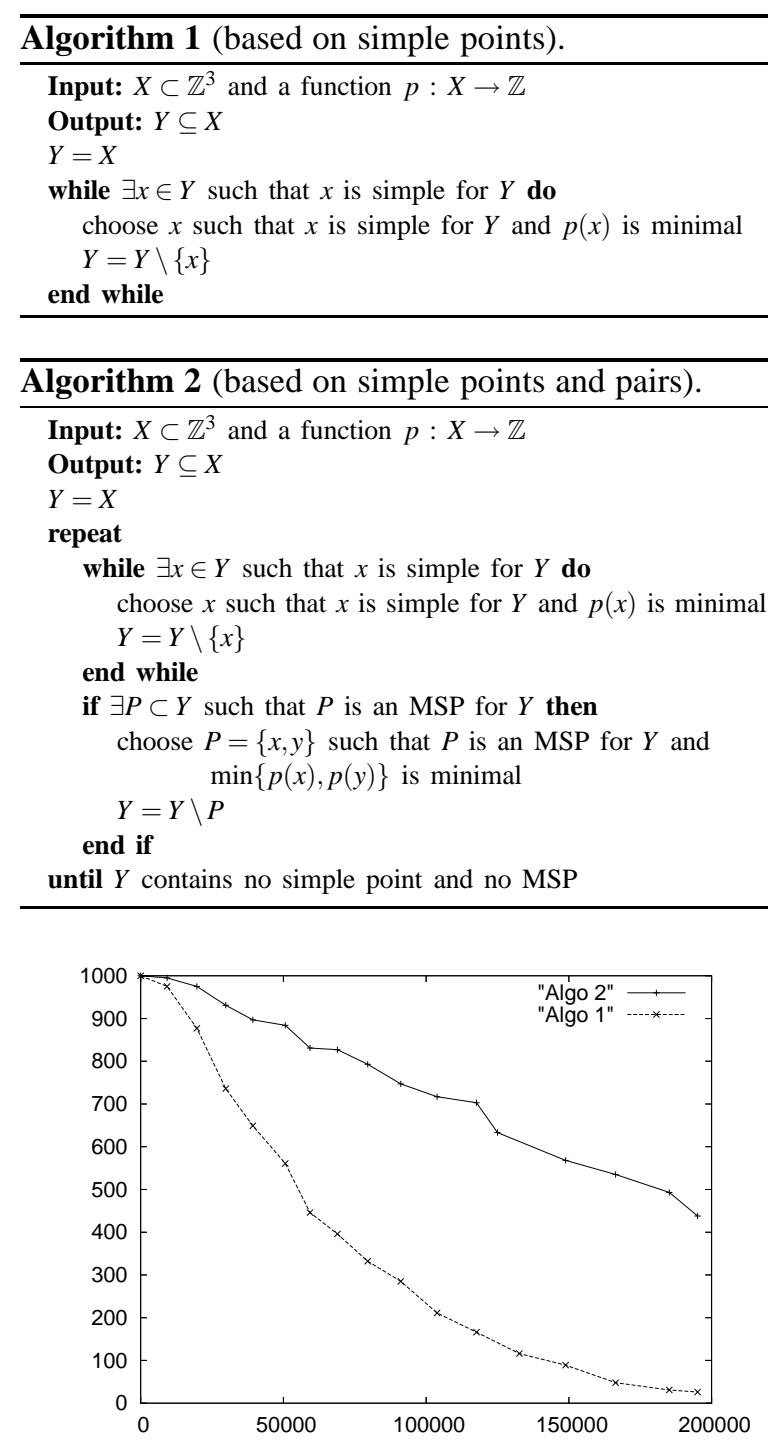

Figure 7. Effectiveness of reduction algorithms based on only simple points (Algo 1) and both simple points and MSPs (Algo 2). Horizontal axis: size (number of voxels) of original objects. Vertical axis: number of objects (over 1000 experiments) which were reduced to a single voxel.

The following properties will be used in the proofs of the properties of Section 5 (see Appendix C).

Lemma 17 Let $F \sqsubseteq \mathbb{F}^{3}$. Let $P \sqsubseteq F$ be a pair, and let $g_{1}, g_{2} \in P^{+}$be the two 3-faces of $P$. Then:

$$
\begin{array}{r}
\operatorname{Att}\left(\hat{g_{1}}, F\right) \cap \operatorname{Att}\left(\hat{g_{2}}, F\right)=\hat{g_{1}} \cap \hat{g_{2}} \\
\operatorname{Att}\left(\hat{g_{1}}, F\right) \cup \operatorname{Att}\left(\hat{g_{2}}, F\right)=\operatorname{Att}(P, F) \cup\left(\hat{g_{1}} \cap \hat{g_{2}}\right)
\end{array}
$$


The proof of the above lemma easily follows from the definitions. The following proposition is elementary.

Proposition 18 Let $B, W, X, Y, Z \preceq \mathbb{F}^{3}$. If $W \cup X=Y \cup$ $Z$ and $B$ is a non-empty connected component of $W$ such that $B \cap Z=\emptyset$ and $B \cap X=\emptyset$, then $B$ is a connected component of $Y$.

Let $F \preceq \mathbb{F}^{3}$, and let $\left\langle F_{0}, \ldots, F_{\ell}\right\rangle$ be a collapse sequence from $F=F_{0}$ to $F_{\ell}$. Let $S=\left\langle\left(f_{1}, g_{1}\right), \ldots,\left(f_{\ell}, g_{\ell}\right)\right\rangle$ be the sequence of pairs of faces of $F$ such that $F_{i}=$ $F_{i-1} \backslash\left\{f_{i}, g_{i}\right\}, i=1, \ldots, \ell$. We also call the sequence $S$ a collapse sequence for $F$. The following proposition gives a condition under which two consecutive pairs can be permuted in a collapse sequence.

Proposition 19 Let $F \preceq \mathbb{F}^{3}$. Let $S=\left\langle\left(f_{1}, g_{1}\right)\right.$, $\left.\left(f_{2}, g_{2}\right)\right\rangle$ be a collapse sequence for $F$. If $g_{2} \nsubseteq f_{1}$, then the sequence $S^{\prime}=\left\langle\left(f_{2}, g_{2}\right),\left(f_{1}, g_{1}\right)\right\rangle$ is also a collapse sequence for $F$.

Proof. We have to prove that i) $\left(f_{2}, g_{2}\right)$ is free for $F$, and that ii) $\left(f_{1}, g_{1}\right)$ is free for $F \backslash\left\{f_{2}, g_{2}\right\}$. By hypothesis $\left(f_{2}, g_{2}\right)$ is free for $F \backslash\left\{f_{1}, g_{1}\right\}$, thus we know that $g_{2} \subseteq f_{2}$ and that $g_{2}$ is not strictly included in any other face of $F \backslash\left\{f_{1}, g_{1}\right\}$. Since we have $g_{2} \nsubseteq f_{1}$, we deduce i). As $\left(f_{2}, g_{2}\right)$ is free for $F \backslash\left\{f_{1}, g_{1}\right\}, f_{1}$ and $g_{1}$ are faces of $F \backslash\left\{f_{2}, g_{2}\right\}$. Since by hypothesis $\left(f_{1}, g_{1}\right)$ is free for $F$, we know that $f_{1}$ is the only face of $F$ which includes $g_{1}$, and thus also the only face of $F \backslash\left\{f_{2}, g_{2}\right\}$ which includes $g_{1}$. Hence ii) holds.

From Proposition 19, we deduce by induction the following proposition. It gives a condition under which a particular pair in a collapse sequence may be deferred to the end of the sequence.

Proposition 20 Let $F \preceq \mathbb{F}^{3}$. Let $i, n \in \mathbb{N}$ be such that $1 \leq i \leq n$, and let $S=\left\langle\left(f_{1}, g_{1}\right), \ldots,\left(f_{i}, g_{i}\right), \ldots,\left(f_{n}, g_{n}\right)\right\rangle$ be a collapse sequence for $F$ such that, for all $j$ in $\{i+1, \ldots, n\}, g_{j} \nsubseteq f_{i}$. Then, the sequence $S^{\prime}=$ $\left\langle\left(f_{1}, g_{1}\right), \ldots,\left(f_{i-1}, g_{i-1}\right),\left(f_{i+1}, g_{i+1}\right), \ldots,\left(f_{n}, g_{n}\right)\right.$, $\left.\left(f_{i}, g_{i}\right)\right\rangle$ is also a collapse sequence for $F$.

Proposition 21 Let $F \sqsubseteq \mathbb{F}^{3}$. Let $g, g^{\prime} \in F^{+}$be such that $\operatorname{dim}\left(\hat{g} \cap \hat{g}^{\prime}\right)=2$. Let $P=\left\{g, g^{\prime}\right\}^{-} \sqsubseteq F$. If $P$ collapses onto $\operatorname{Att}(P, F)$ and $\operatorname{Att}(\hat{g}, F)=\hat{g}^{*}$, then $\hat{g}^{\prime}$ collapses onto Att $\left(\hat{g}^{\prime}, F\right)$.

Proof. Let $S=\left\langle\left(f_{i}, g_{i}\right)\right\rangle_{i=1}^{n}(n \geq 1)$ be a collapse sequence from $P$ onto $\operatorname{Att}(P, F)$. We necessarily have $\operatorname{dim}\left(f_{1}\right)=3$. Moreover, $f_{1} \neq g$, since for all $f \in \hat{g}^{*}$, $f \in \operatorname{Att}(P, F)$ or $f \subseteq g^{\prime}$, as a consequence of Lemma 17 (2). Consequently, $f_{1}=g^{\prime}$. Let $P^{\prime}=P \backslash\left\{f_{1}, g_{1}\right\}$. The sequence $S=\left\langle\left(f_{i}, g_{i}\right)\right\rangle_{i=2}^{n}$ is a collapse sequence from
$P^{\prime}$ onto $\operatorname{Att}(P, F)$. There must exist $k \in\{2, \ldots, n\}$ such that $f_{k}=g$. It can be easily seen that for all $i \in[k+1, n]$, since $\operatorname{Att}(\hat{g}, F)=\hat{g}^{*}$ and in view of Lemma 17 (2), $g_{i} \nsubseteq g=f_{k}$. Thus, from Proposition 20 , the sequence $\left\langle\left(f_{2}, g_{2}\right), \ldots,\left(f_{k-1}, g_{k-1}\right)\right.$, $\left.\left(f_{k+1}, g_{k+1}\right), \ldots,\left(f_{n}, g_{n}\right),\left(f_{k}, g_{k}\right)\right\rangle$ is a collapse sequence from $P^{\prime}$ onto $\operatorname{Att}(P, F)$, and then, $S^{\prime}=$ $\left\langle\left(f_{1}, g_{1}\right),\left(f_{2}, g_{2}\right), \ldots, \quad\left(f_{k-1}, \quad g_{k-1}\right), \quad\left(f_{k+1}, g_{k+1}\right)\right.$, $\left.\ldots,\left(f_{n}, g_{n}\right)\right\rangle$ is a collapse sequence from $P$ onto $\operatorname{Att}(P, F) \cup\left\{f_{k}, g_{k}\right\}=\operatorname{Att}(P, F) \cup\left\{g, g \cap g^{\prime}\right\}$. It can be easily seen that $S^{\prime}$ is also a collapse sequence from $\hat{g}^{\prime}$ onto $\operatorname{Att}\left(\hat{g}^{\prime}, F\right)$.

The following theorem has been proved by Chillingworth in the framework of simplicial complexes. Both the result and its proof easily extend to cubical complexes.

Theorem 22 (Adapted from Chillingworth [8])

Let $P \sqsubseteq \mathbb{F}^{3}$ be a topological ball, embedded rectilinearly as a convex subset of the Euclidean 3-space. Let $G$ be a subcomplex of $\operatorname{Att}\left(P, \mathbb{F}^{3}\right)$ such that $|C[G]|=$ $\chi(G)=1$ and $G \neq \operatorname{Att}\left(P, \mathbb{F}^{3}\right)$. Then, $P$ collapses onto $G$.

\section{Appendix C: Proofs of the results of Section 5}

Proof of Proposition 7. Suppose that $|C[P]| \neq 1$; thus $|C[P]|=2$. Let $g_{1}, g_{2} \in P^{+}$be the two 3-faces of $P$. Then $g_{1}$ and $g_{2}$ are not adjacent, i.e. $\hat{g_{1}} \cap \hat{g_{2}}=\emptyset$. From Lemma 17, $\operatorname{Att}\left(\hat{g_{1}}, F\right) \cap \operatorname{Att}\left(\hat{g_{2}}, F\right)=\emptyset$ and $\operatorname{Att}(P, F)=\operatorname{Att}\left(\hat{g_{1}}, F\right) \cup \operatorname{Att}\left(\hat{g_{2}}, F\right)$. Consequently, the fact that $P$ collapses onto $\operatorname{Att}(P, F)$ implies that $\hat{g_{1}}$ collapses onto $\operatorname{Att}\left(\hat{g_{1}}, F\right)$ and that $\hat{g_{2}}$ collapses onto $\operatorname{Att}\left(\hat{g_{2}}, F\right)$. But then, $\hat{g_{1}}$ and $\hat{g_{2}}$ are simple voxels for $F$, which is contradictory with the definition of an MSP.

Proof of Proposition 8. Let $g_{1}, g_{2} \in P^{+}$be the two 3 -faces of $P$. From Proposition 7, we have $|C[P]|=$ 1 , thus $\hat{g_{1}} \cap \hat{g_{2}} \neq \emptyset$. From Lemma 17 (1) we have $\hat{g_{1}} \cap \hat{g_{2}} \subseteq \operatorname{Att}\left(\hat{g_{1}}, F\right)$, thus $\left|C\left[\operatorname{Att}\left(\hat{g_{1}}, F\right)\right]\right| \geq 1$.

Suppose now that $\left|C\left[\operatorname{Att}\left(\hat{g_{1}}, F\right)\right]\right|>1$. Since $\hat{g_{1}} \cap \hat{g_{2}} \subseteq$ $\operatorname{Att}\left(\hat{g_{1}}, F\right)$, one connected component $A$ of $\operatorname{Att}\left(\hat{g_{1}}, F\right)$ includes $\hat{g_{1}} \cap \hat{g_{2}}$, and there exists another connected component $B$ of $A t t\left(\hat{g_{1}}, F\right)$ such that $B \cap\left(\hat{g_{1}} \cap \hat{g_{2}}\right)=\emptyset$, hence $B \cap \hat{g_{2}}=\emptyset$ (since $B \subseteq \hat{g_{1}}$ ). From Lemma 17 (2) and Proposition 18, $B$ is also a connected component of $\operatorname{Att}(P, F)$. From Proposition 7 and since collapse preserves the number of connected components, we 
have $|C[\operatorname{Att}(P, F)]|=1$. Hence, $\operatorname{Att}(P, F)=B$. Let $A^{\prime}=A \backslash\left(\hat{g_{1}} \cap \hat{g_{2}}\right)$. Note that $A^{\prime} \subseteq \operatorname{Att}(P, F)=B$ and that $A^{\prime} \cap B=\emptyset$ (since $A \cap B=\emptyset$ ); thus $A^{\prime}=\emptyset$, and hence $A=\hat{g_{1}} \cap \hat{g_{2}}$. Since $\operatorname{Att}(P, F) \cap \hat{g_{2}}=B \cap \hat{g_{2}}=\emptyset$, from Lemma 17 (2) we must have $A=\hat{g_{1}} \cap \hat{g_{2}}=$ $\operatorname{Att}\left(\hat{g_{2}}, F\right)$, and obviously $\hat{g_{2}}$ collapses onto $\operatorname{Att}\left(\hat{g_{2}}, F\right)$. The voxel $\hat{g_{2}}$ is then simple for $F$, which is contradictory. Therefore $\left|C\left[\operatorname{Att}\left(\hat{g_{1}}, F\right)\right]\right|=1$, and the same reasoning holds for $g_{2}$.

Proof of Proposition 9. Let $g_{1}, g_{2} \in P^{+}$be the two 3-faces of $P$. Suppose that $\operatorname{Att}\left(\hat{g_{1}}, F\right)={\hat{g_{1}}}^{*}$. By definition, $P$ collapses onto $\operatorname{Att}(P, F)$. Since $g_{1} \notin \operatorname{Att}(P, F)$, any collapse sequence from $P$ to $\operatorname{Att}(P, F)$ involves a free pair $\left(g_{1}, h\right)$, with $h \in \hat{g_{1}}{ }^{*}$. Then $h \in \operatorname{Att}\left(\hat{g_{1}}, F\right)$ and $h \notin \operatorname{Att}(P, F)$. From Lemma 17 (2), $h \in \hat{g_{1}} \cap \hat{g_{2}}$ (i.e., $h \subseteq g_{1} \cap g_{2}$ ), and from the very definition of a free pair, $\operatorname{dim}(h)=\operatorname{dim}\left(g_{1}\right)-1=2$. Consequently, we have $g_{1} \cap g_{2}=h$. From Proposition 21, we know that $\hat{g_{2}}$ collapses onto $\operatorname{Att}\left(\hat{g_{2}}, F\right)$, i.e., $\hat{g_{2}}$ is a simple voxel for $F$, which is contradictory.

Proof of Lemma 12. Let $g_{1}, g_{2} \in P^{+}$be the two 3faces of $P$. From Lemma 17 (2), we have $\chi(\operatorname{Att}(P, F) \cup$ $\left.\left(\hat{g_{1}} \cap \hat{g_{2}}\right)\right)=\chi\left(\operatorname{Att}\left(\hat{g_{1}}, F\right) \cup \operatorname{Att}\left(\hat{g_{2}}, F\right)\right)$ which leads to $\chi(\operatorname{Att}(P, F))+\chi\left(\hat{g_{1}} \cap \hat{g_{2}}\right)-\chi\left(\operatorname{Att}(P, F) \cap \hat{g_{1}} \cap\right.$ $\left.\hat{g_{2}}\right)=\chi\left(\operatorname{Att}\left(\hat{g_{1}}, F\right)\right)+\chi\left(\operatorname{Att}\left(\hat{g_{2}}, F\right)\right)-\chi\left(\operatorname{Att}\left(\hat{g_{1}}, F\right) \cap\right.$ $\left.\operatorname{Att}\left(\hat{g_{2}}, F\right)\right)$. From Lemma 17 (1), we then have $\chi(\operatorname{Att}(P, F))+2 \chi\left(\hat{g_{1}} \cap \hat{g_{2}}\right)-\chi\left(\operatorname{Att}(P, F) \cap \hat{g_{1}} \cap \hat{g_{2}}\right)=$ $\chi\left(\operatorname{Att}\left(\hat{g_{1}}, F\right)\right)+\chi\left(\operatorname{Att}\left(\hat{g_{2}}, F\right)\right)$. From Proposition 10, $\chi\left(\operatorname{Att}\left(\hat{g_{1}}, F\right)\right) \leq 0$ and $\chi\left(\operatorname{Att}\left(\hat{g_{2}}, F\right)\right) \leq 0$. Moreover, as $P$ collapses onto $\operatorname{Att}(P, F)$, we necessarily have $\chi(\operatorname{Att}(P, F))=\chi(P)=1$, and we obviously have $\chi\left(\hat{g_{1}} \cap \hat{g_{2}}\right)=1$. Consequently, we obtain $1+2 \times 1-\chi\left(\operatorname{Att}(P, F) \cap \hat{g_{1}} \cap \hat{g_{2}}\right) \leq 0$, i.e. $\chi\left(\operatorname{Att}(P, F) \cap \hat{g_{1}} \cap \hat{g_{2}}\right) \geq 3$.

\section{Proof of Proposition 15.}

" $\Rightarrow$ " side of the proof. We suppose that $P$ is an MSP for $F$. Conditions 1, 2, 3, 4, and 5 follow from Propositions $11,8,10,13$, and 14 , respectively.

" $\Leftarrow$ " side of the proof. Suppose that $1,3,4$ and 5 hold. Proof of simplicity. Let $d P=\operatorname{Att}\left(P, \mathbb{F}^{3}\right)$. We cannot have $\operatorname{Att}(P, F)=d P$, otherwise 5 would not hold. Let $h$ be a 2 -face in $d P \backslash \operatorname{Att}(P, F)$. Then Theorem 22 tells us that $d P \backslash\{h\}$ collapses onto $\operatorname{Att}(P, F)$. This implies that $P$ collapses onto $\operatorname{Att}(P, F)$, since $P$ evidently collapses onto $d P \backslash\{h\}$. So it follows from Proposition 3 that $P$ is simple for $F$.

Proof of minimality. From Theorem 6, if a voxel $\hat{g}$ is simple for $F$ then $\chi(\operatorname{Att}(\hat{g}, F))=1$. Thus from Condition 3 , for any $g \in P^{+}, \hat{g}$ is not a simple voxel for $F$.

\section{Appendix D: Lumps and simple sets}

Let us consider the guided homotopic thinning procedure described in Section 1. When performing such a procedure, the result is expected to fulfill a property of minimality. By construction, the result $X_{n}$ is minimal in the sense that it contains no simple point outside of $K$. However, we could formulate a stronger minimality requirement, which seems natural for this kind of transformation: informally, the result $X_{n}$ should not strictly include any set $Y$ which is "topologically equivalent" to $X$, and which contains $K$. We say that a homotopic skeleton of $X$ constrained by $K$ is globally minimal if it fulfils this condition.

Now, a fundamental question arises: is any homotopic skeleton globally minimal? Let us illustrate this problem in dimensions 2 and 3 . In $\mathbb{Z}^{2}$, consider a full rectangle $X$ of any size, and the constraint set $K=$ $\emptyset$. Obviously, this object $X$ is topologically equivalent to a single point, thus only homotopic skeletons which are singletons are globally minimal. A. Rosenfeld proved in [24] that any homotopic skeleton of $X$ is indeed reduced to a single point.

However, in dimension 3 , this property does not hold: if $X$ is $e$.g. a full $10 \times 10 \times 10$ cube, we may find a homotopic skeleton of $X$ (with empty constraint set) which is not reduced to a single point. A classical counter-example is Bing's house with two rooms [6], illustrated in Figure 8. One can enter the lower room of the house by the chimney passing through the upper room, and vice-versa. A discrete version $B$ of Bing's house is displayed in Figure 9. It can be seen that Bing's house can be carved from a full cube by iterative removal of simple points. It can also be seen that $B$ contains no simple point: deleting any point from $B$ would create a "tunnel".

It could be argued that objects like Bing's house are unlikely to appear while processing real (noisy) images, because of their complex shape and their size. However, we found that there exists a large class of objects presenting similar properties, some of them being quite small (less than 50 voxels). Let us call a lump relative to $K$ any object $X$ which has no simple 


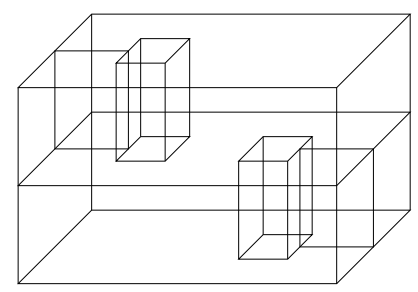

Figure 8. Bing's house with two rooms.
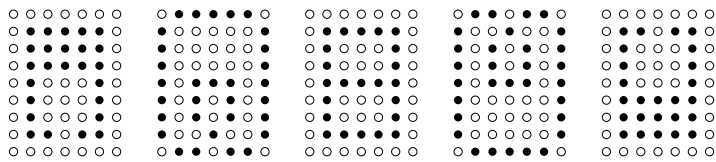

Figure 9. A discrete version of Bing's house, decomposed into its five planar slices for visualisation. The 26-adjacency relation is used for object points. This object is made of 135 voxels.

point outside of $K$, and which strictly includes a subset $Y$ containing $K$ and topologically equivalent to $X$ (i.e., a homotopic skeleton which is not globally minimal). More formally, a lump can be defined as follows, thanks to the notion of simple-equivalence.

Definition 23 Let $F, G \sqsubseteq \mathbb{F}^{3}$. We say that $F$ and $G$ are simple-equivalent if there exists a sequence of complexes $\left\langle F_{0}, \ldots, F_{\ell}\right\rangle$ such that $F_{0}=F, F_{\ell}=G$, and for any $i \in\{1, \ldots, \ell\}$, we have either

i) $F_{i}=F_{i-1} \bigcirc x_{i}$, where $x_{i}$ is a voxel which is simple for $F_{i-1}$; or

ii) $F_{i-1}=F_{i} \ominus x_{i}$, where $x_{i}$ is a voxel which is simple for $F_{i}$.

Definition 24 Let $G \sqsubseteq F \sqsubseteq \mathbb{F}^{3}$ be such that $F$ and $G$ are simple-equivalent. If $F \neq G$ and $F$ does not contain any simple voxel outside $G$, then we say that $F$ is a lump relative to $G$, or simply a lump.

For example, the Bing's house of Figure 9 is a lump (relative to any one of its voxels), which is composed of 135 voxels (represented by black dots). Also, the configurations displayed in Figures 4 and 5 are lumps, made respectively of 32 and 14 voxels.

An even smaller example of a lump is shown in Figure 10a (see in Figure 11 some steps of a sequence which shows that $10 \mathrm{a}$ and $10 \mathrm{~b}$ are simple-equivalent). The complex of Figure 10a is a lump made of 11 voxels. It can be seen that, contrarily to the two previous examples, this lump contains no simple set. A variant of this configuration, found by J. Chaussard [7], is displayed in Figure 12a: it is a lump made of only 10 voxels. We conjecture that 10 is the smallest possible size for a lump.

Remark 25 The existence of lumps that contain no simple set leads us to consider the following generalization of the notion of simple set. A subcomplex $G \sqsubseteq F$ is called SE-simple for $F$ (where SE stands for Simple-Equivalence) if $F$ and $F \odot G$ are simpleequivalent. For example, the voxel $x$ in the complex $F$ of Figure 10a is SE-simple for F, although it is not a simple voxel for $F$. (This kind of configuration has previously been considered [21,13]). Of course, any simple set is SE-simple, and the preceding example proves that the converse is not true in general. However, it is not possible to characterise locally, in the manner of Proposition 3, a voxel or a set which is SEsimple: whereas $x$ is $S E$-simple for the complex $F$ of Figure 10a, $x$ is not $S E$-simple for the complex $F^{\prime}$ that is given by the union of $x$ and the six other voxels in $F$ that intersect $x$, even though Att $\left(x, F^{\prime}\right)=\operatorname{Att}(x, F)$.

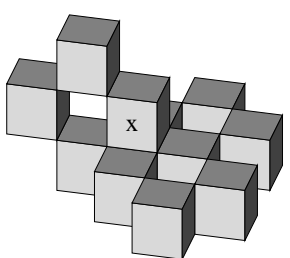

(a)

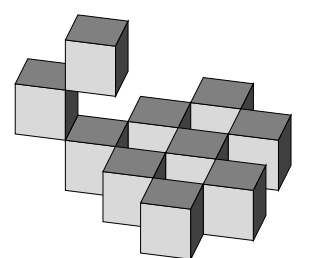

(b)
Figure 10. A lump made of 11 voxels is depicted in (a). It contains no simple voxel, and is simple-equivalent to the complex in (b), made of 10 voxels. Both objects have three tunnels.

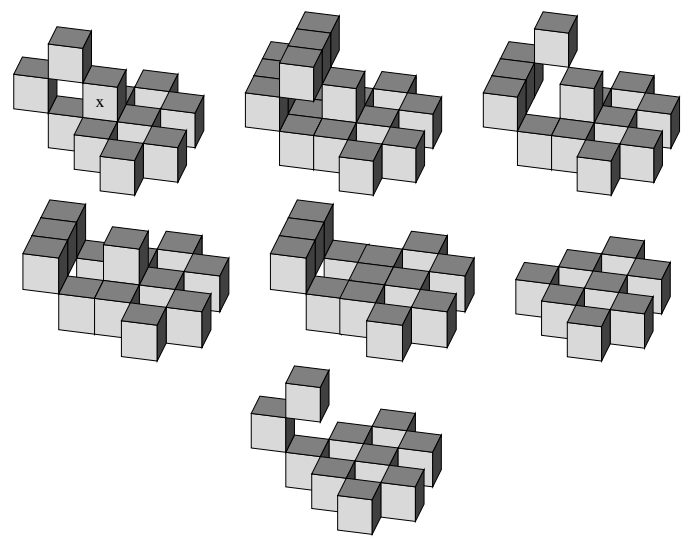

Figure 11. Some steps of a sequence showing the simple-equivalence between the objects of Figure 10. 


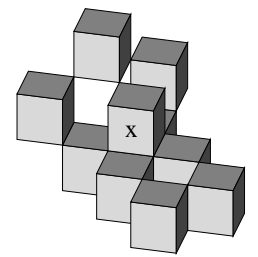

(a)

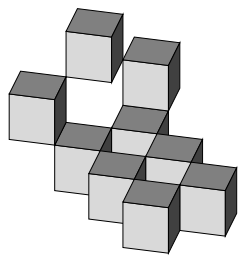

(b)

Figure 12. A lump made of 10 voxels is depicted in (a). It contains no simple voxel, and is simple-equivalent to the complex in (b). 